\title{
Strong X-ray Bursts with Expansion: Oscillations due to Sound Waves in Atmospheres
}

\author{
Iosif Lapidus ${ }^{1 \star}$, Luciano Nobili ${ }^{2}$, Roberto Turolla ${ }^{2}$ \\ 1 Institute of Astronomy, University of Cambridge, \\ Madingley Road, Cambridge CB3 OHA, UK \\ ${ }^{2}$ Department of Physics, University of Padova, Via Marzolo 8, 35131 Padova, Italy
}

\section{Oscillations in $\mathrm{X}$-ray bursts with expansion}

Photospheric expansion has been observed during several powerful type I Xray bursts, and it is associated with a supersonic outflow driven by the superEddington energy release in the thermonuclear He-burning at the base of the envelope (Lewin et al. 1993). Such expanded atmospheres represent perfect background for the propagation of sound, and we believe there is a number of arguments in favor of the reality of these phenomena.

We have suggested an acoustic origin for the second range photospheric oscillations detected in the X-ray bursters 1608-522 and 2127+119 (Lapidus et al. 1994). On the basis of our models of radiation driven winds from neutron stars during strongest bursts (Nobili et al. 1994), we have shown that purely radial standing sound waves in expanded spherical envelopes can have periods very close to the observed ones. Both the photosphere and the sonic surface may act as a reflecting boundary; this is the reason of quite different periods in the two sources ( $0.65 \mathrm{~s}$ and $\sim 7 \mathrm{~s}$, respectively).

Although the physics of type II bursts from the Rapid Burster is somewhat different, some of them, too, show evidence of photospheric expansion. The QPO discovered in type II bursts, are quite dissimilar to those observed in many bright LMXBs (Lewin et al. 1993). The centroid frequencies range from $\sim 2$ to $\sim 7 \mathrm{~Hz}$, being strongly anticorrelated with the peak flux in the burst.

The QPO in type II bursts may also have an acoustic origin. The structure of the shell is thought to be similar to that of our models of the final part of the expansion/contraction phase. The frequency of the principal mode of the "photospheric" standing wave is $\approx 8 \mathrm{~Hz}$ for the model with the lowest possible $M_{\text {env }}$, while a frequency $\approx 2 \mathrm{~Hz}$ corresponds to the eigenfrequency in an atmosphere with $\dot{M} \sim 15-20 \dot{M}_{E}$. The sound wave frequency is higher for weaker bursts, and this is indeed consistent with the observed anticorrelation of the QPO frequency with the average burst peak flux.

* The Royal Astronomical Society Sir Norman Lockyer Fellow 


\section{Oscillations in the AqI X-1 burst}

Coherent $7.6 \mathrm{~Hz}$ oscillations were once detected during a type I burst of Aql X-1 (Schoelkopf \& Kelley 1991). They were detected only in the 80 s portion of the light curve around the peak of the burst, and they were interpreted as reflecting the proper rotation of the underlying neutron star. In our opinion this explanation is highly implausible. The reason is that after first few seconds of the burst the burning must be completely uniform. The time periods between successive bursts on Aql X-1 are very high, so as the burst starts at some location on the star, the burning front propagates with the convective speed $\gtrsim 10^{6}$ $\mathrm{cm} / \mathrm{s}$ (Bildsten 1995). In $\lesssim 10 \mathrm{~s}$ the whole NS surface will be burning simultaneously. The asymmetry of the burst may not be due to nonuniform distribution of the nuclear fuel either. The magnetic field cannot be high enough $\left(\gtrsim 10^{10}\right.$ G) to sustain such asymmetries. Should it happen, such oscillations would be observed not only in the burst but in the persistent flux as well, which is not the case. In the absence of external forces the only reason for asymmetries may be random inhomogeneities. At temperatures $\sim(1-10) \times 10^{8} \mathrm{~K}$ and proton thermal velocities $v_{p} \sim(1-3) \times 10^{8} \mathrm{~cm} / \mathrm{s}$ the latter ones will be disappearing on the hydrodynamical time scale $t_{\text {hyd }} \sim R_{\text {ns }} / v_{\mathrm{p}} \lesssim 10^{-2} \mathrm{~s}$, while the $7.8 \mathrm{~Hz}$ oscillations were claimed to persist for tens of seconds.

We propose that these oscillations also may be due to radial standing sound waves. Due to poor time resolution of Einstein data the photospheric expansion of the envelope can be neither excluded nor confirmed, while the peak luminosity is consistent with the Eddington limit, $\sim 7 \times 10^{-8} \mathrm{erg} /\left(\mathrm{cm}^{2} \mathrm{~s}\right)$ at a distance $D=4$ kpc (the most plausible value cited). Assuming that an expansion did occur, the interpretation of oscillations becomes straightforward. The main modes of sound waves have frequencies $<12 \mathrm{~Hz}$, and the $7.6 \mathrm{~Hz}$ oscillations may be easily reproduced. Furthermore, standing waves are the eigenmodes, i.e. they are very coherent, in accordance with observations. The main mode frequency almost does not depend on parameters of the expanded atmosphere in the relevant range, i.e. not far from the end of expansion/contraction phase, so this frequency will remain constant along the burst.

\section{References}

Bildsten L., 1995, ApJ (to appear; Caltech preprint)

Lapidus I., Nobili L., Turolla R., 1994, ApJ 431, L103

Lewin W.H.G., Van Paradijs J., Taam R.E., 1993, Space Sci. Rev. 62, 223

Nobili L., Turolla R., Lapidus I., 1994, ApJ 433, 276

Schoelkopf R.J., Kelley R.L., 1991, ApJ 375, 696 